\title{
DEPRESSÃO E ENVELHECIMENTO SOB A PERSPECTIVA MULTIPROFISSIONAL: UMA REVISÃO DE LITERATURA
}

\author{
DEPRESSION AND AGING: A SYSTEMATIC REVIEW.
}

\author{
José Ewayr Mariano de Araújo ${ }^{1}$ \\ Júlia Ramos Vieira ${ }^{2}$ \\ Lucenildo Laerty da Silva Sales ${ }^{3}$ \\ Mirelle de Alcântara Martins Macedo ${ }^{4}$
}

\begin{abstract}
RESUMO: OBJETIVO: Identificar e analisar produções científicas que apontem o manejo multiprofissional sobre a depressão na terceira idade, bem como o uso de medicamentos e estado nutricional do idoso e a influencia destes na qualidade de vida METODOLOGIA: Este é um estudo exploratório do tipo revisão de literatura. Foram analisados 20 artigos científicos, em língua portuguesa, entre os anos 20152019, publicados na íntegra nas bases de dados Literatura Latino-Americana e do Caribe em Ciências da Saúde (LILACS), Scientific Eletronic Library Online (SciELO) e Biblioteca Virtual em Saúde (BVS). Destes, seis artigos foram selecionados, sendo considerados como mais relevantes e que abordassem a abordagem multidisciplinar, trazendo aspectos psicológicos, farmacêuticos e nutricionais existentes entre a depressão e o envelhecimento. RESULTADOS E DISCUSSÃO: O idoso encontrase em vulnerabilidade do ponto de vista biopsicossocial, uma vez que a perda da autonomia e do papel social a prevalência de doenças crônicas reflete na redução da qualidade de vida, uso excessivo de medicamentos e alterações no estado nutricional. Os estudos apontam que o sexo feminino é mais acometido por estes agravos e que as equipes de saúde (especialmente as de atenção básica) têm buscado ferramentas para contornar estas condições. CONCLUSÃO: A depressão está relacionada com a baixa qualidade de vida no idoso e interfere no estado nutricional e no padrão medicamentoso utilizado por eles, debilitando ainda mais seu estado geral e gerando situações de dependência e incapacidade, evidenciando sintomas depressivos.
\end{abstract}

\footnotetext{
${ }^{1}$ Farmacêutico. Farmacêutico. Farmacologia clínica e atenção farmacêutica. Mestrando em Saúde Pública - PPGSP/UEPB.

${ }^{2}$ Psicóloga. Especialista em Saúde Mental. Mestranda em Saúde Pública - PPGSP/UEPB.

${ }^{3}$ Enfermeiro. Especialista em Gestão e Auditoria em Serviços de Saúde. Mestrando em Saúde Pública - PPGSP/UEPB.

${ }^{4}$ Nutricionista. Mestranda em Saúde Pública - PPGSP/UEPB.
} 
Palavras chave: Depressão. Psicotrópicos. Qualidade de vida. Estado nutricional. Idoso.

ABSTRACT: OBJECTIVE: To identify and analyze scientific productions that point to or manage multiprofessional depression in the elderly, as well as the use of medications and the nutritional status of the elderly and influences the quality of life. METHODOLOGY: This is an exploratory study of the literature review type. Twenty scientific articles were analyzed, in Portuguese, between the years 2015 and 2019, published in full in the databases Literatura Latino-Americana e do Caribe em Ciências da Saúde (LILACS), Scientific Eletronic Library Online (SciELO) e Biblioteca Virtual em Saúde (BVS).. Destinations, six articles were selected, being considered the most relevant and addressing a multidisciplinary approach, bringing psychological, pharmaceutical and nutritional aspects existing between a depression and an illness. RESULTS AND DISCUSSION: The elderly are vulnerable from the biopsychosocial point of view, since the loss of autonomy and the social role are affected by the prevalence of chronic diseases, reducing quality of life, using drugs excessively and changes in nutritional status. Studies show that females are more affected by these diseases and that health teams (especially primary care) have tools used to get around these conditions. CONCLUSION: Depression is qualified with a low quality of life and does not interfere with the nutritional status and pattern of medications used by them, further weakening their general condition and generating situations of dependence and disability, evidence of depression.

Keywords: Depression. Psychotropic drugs. Quality of life. Nutritional status. Aged. 


\section{INTRODUÇÃO}

O envelhecimento humano é marcado por diversas modificações, essas que podem determinar a progressiva perda de adaptação à novos ambientes e situações. Mudanças físicas, psicológicas e sociais, estão presentes como todas as fases da vida, cada uma com sua singularidade. Com a evolução da sociedade industrial, a valorização do ser humano passou a ser associado à sua produtividade, e à sua contribuição para a sociedade, deixando, o envelhecimento, de ser visto como situação natural, para ser encarado como fenômeno influenciado pela cultura. (UCHOA, 2013).

A Organização Mundial da Saúde (OMS) define envelhecimento como "aquele indivíduo com 60 anos de idade ou mais, limite este válido apenas para os países em desenvolvimento, como o Brasil, pois nos países desenvolvidos admite-se um ponto de corte de 65 anos de idade.

Estudos mostram que o processo de envelhecer está associado com drástica redução da qualidade de vida na população idosa em geral. Como preceito do bemestar biopsicossocial, o envelhecimento ativo tem como fundamento garantir a autonomia.

O envelhecimento ativo objetiva garantir melhor qualidade de vida à pessoa idosa, oportunizando-as e inserindo-as de forma integral no contexto social. $\mathrm{O}$ termo envelhecimento ativo e bem-sucedido tem como princípio a vivencia de uma velhice produtiva e bem vivida, ou seja, envelhecer com saúde e atividade, relacionando-se socialmente. (OMS, 2005).

Em 2025, o Brasil será o $6^{\circ}$ país do mundo com o maior número de idosos, e isso se dá pela transição demográfica em que vivemos, contexto que aponta uma longevidade da população em detrimento dos nascimentos. Entretanto, longevidade, nem sempre é sinônimo de qualidade de vida. Muitos idosos apresentam dependência funcional, declínio emocional e depressão, disfunções orgânicas e dependência medicamentosa. Atrelado à dependência do idoso surge a internação 
de longa duração do idoso, em casas de repouso, excluindo-o de seu contexto e em certa medida, o privando da autonomia e da funcionalidade. Um dos efeitos do avanço da ciência e da tecnológica remete a busca por uma melhor qualidade de vida, e autonomia do sujeito, e dessa forma, surgem programas e atividades visando recuperar o desejo de viver e o investimento em projetos na vida. (QUINALHA, 2010).

Os idosos são o grupo etário campeão na utilização de psicofármacos para tratamento de comorbidades psíquicas e somáticas. O uso destes medicamentos estão associados à situações adversas, como prejuízo cognitivo, delírio e depressão, sendo este último o fator mais fortemente associado ao uso destes medicamentos. (ABI-ACKEL, 2017).

A expectativa de vida elevada e o aumento do envelhecimento da população nos dez últimos anos refletem na maior prevalência de doenças crônicas nãotransmissíveis, deixando o idoso predisponente à polifarmácia, que consiste no de mais de cinco medicamentos concomitantemente e, consequentemente, susceptível à efeitos adversos dos medicamentos, bem como a prescrição desnecessária. (DONIS, 2017; VERA, 2017; NÓBREGA, 2005).

A dependência medicamentosa é frequente e está associada ao panorama de adoecimento da pessoa idosa, que se deve à elevada prevalência de doenças crônicas não- transmissíveis (DCNT) e das sequelas que acompanham o idoso com o avançar da idade. A indústria farmacêutica e as estratégias de marketing utilizadas por ela, além do medicalização imposta pela prática dos profissionais de saúde contribuem para a acentuação deste cenário. (SECOLI, 2010).

Outrossim, a exposição frequente do idoso à polifarmácia leva à questões relevantes sobre a segurança do uso de medicamentos e às prováveis interações medicamentosas que podem acontecer. A gravidade dessas possíveis interações estão relacionadas com as propriedades dos medicamentos e a finalidade de utilização dos mesmos, sendo mais prevalentes na associação de medicamentos utilizados no tratamento de transtornos mentais com medicamentos para outras condições clínicas. (CROSS, 2019; DEKKERS, 2018).

O processo de envelhecer também exerce influência direta sobre o estado nutricional do indivíduo. Isso decorre das alterações fisiológicas normais para a 
situação cronológica, como a redução na quantidade de papilas gustativas, diminuição do olfato e da visão, além da baixa produção de secreção gástrica e saliva e ineficácia da mastigação pela diminuição do número de dentes ou da má adaptação de próteses, além das limitações intestinais. (GARCIA; ROMANI; LIRA, 2007).

Os idosos estão mais propensos a desenvolverem problemas de ordem nutricional por consequência das alterações fisiológicas e sociais, além do uso de medicamentos diversos, redução na qualidade da alimentação, depressão e outros transtornos somáticos e psíquicos. (ACUÑA, 2004).

A depressão tem um papel decisivo na condição nutricional do idoso. A perda de amigos, o isolamento e a sensação de abandono, a redução da autonomia e as dificuldades associadas ao autocuidado reforçam a presença dos quadros depressivos nessa população, levando ao desinteresse pelas atividades diárias, que inclui a preparo das refeições e a alimentação. (GUEDES; GAMA; TIUSSI, 2008).

Não sendo tratada adequadamente, a depressão associa-se à elevação na morbimortalidade e maiores custos assistenciais, impactando negativamente na qualidade de vida, no consumo alimentar e no ganho de peso. (LANGA, 2004; GERMAN, 2008; YOSHIMURA, 2013).

Assim, a equipe multiprofissional de saúde tem um papel muito importante no que tange a reinserção social do idoso, pois, através do cuidado holístico e integral reposiciona essa população em sua comunidade e em seu contexto familiar, de forma digna e prazerosa, resgatando a qualidade de vida destes grupos.

Portanto, o objetivo deste estudo é realizar uma busca de trabalhos publicados na íntegra sobre os casos de depressão na pessoa idosa, apontando as suas causas e destacar as intervenções relacionadas ao envelhecimento ativo que buscam reduzir esse quadro. 


\section{METODOLOGIA}

Este é um estudo exploratório do tipo revisão de literatura. Foram analisados 20 artigos científicos, em língua portuguesa, entre os anos 2015-2019, publicados na íntegra nas bases de dados Literatura Latino-Americana e do Caribe em Ciências da Saúde (LILACS), Scientific Eletronic Library Online (SciELO) e Biblioteca Virtual em Saúde (BVS). Destes, seis artigos foram selecionados de acordo com os descritores do DeCS (Descritores em Ciências da Saúde), "Depressão", "Psicotrópicos", "Qualidade de Vida", "Nutrição" e "Idosos", captando as pesquisas consideradas mais relevantes e que abordassem a intervenção em sua totalidade, trazendo seus aspectos físicos e psicológicos existentes entre a depressão e o envelhecimento.

O presente estudo limitou-se apenas às publicações consideradas de livre acesso nas bases de dados citadas anteriormente. A partir disso, somente os artigos com resumo que contemplasse o assunto a ser estudado foram utilizados nesta pesquisa.

\section{RESULTADOS}

Ao final, foram incluídos 6 estudos para compor esta revisão de literatura. A Tabela 1 apresenta a distribuição por ano e país de origem, título, autores e local de publicação. 
Tabela 1: Artigos selecionados para análise.

\begin{tabular}{|c|c|c|}
\hline $\begin{array}{c}\text { Ano de } \\
\text { publicaçãol } \\
\text { País de origem }\end{array}$ & Título do artigo & $\begin{array}{l}\text { Local de } \\
\text { Publicação }\end{array}$ \\
\hline Brasil, 2018. & $\begin{array}{|ll|}\text { Envelhecimento eSperli Geraldes Marin Sasaki, } & \text { Camila Garcel Pancote, Natália } \\
\text { uso } & \text { deMaria de Lourdes Sperli } \\
\text { psicotrópicos } & \begin{aligned} \text { Geraldes Santos, Zaida Aurora } \\
\text { Sperli Geraldes Soler. }\end{aligned} \\
\end{array}$ & $\begin{array}{l}\text { Revista } \\
\text { Enfermagem } \\
\text { Brasil }\end{array}$ \\
\hline Brasil, 2017. & 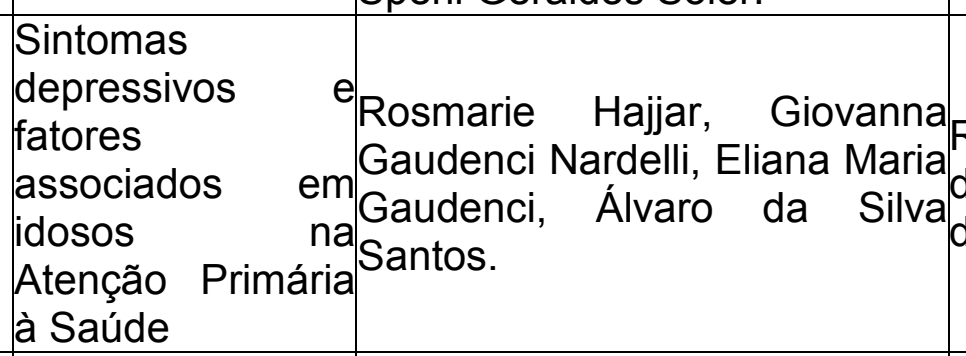 & $\begin{array}{l}\text { Revista da Red } \\
\text { de Enfermager } \\
\text { do Nordeste }\end{array}$ \\
\hline Brasil, 2018. & 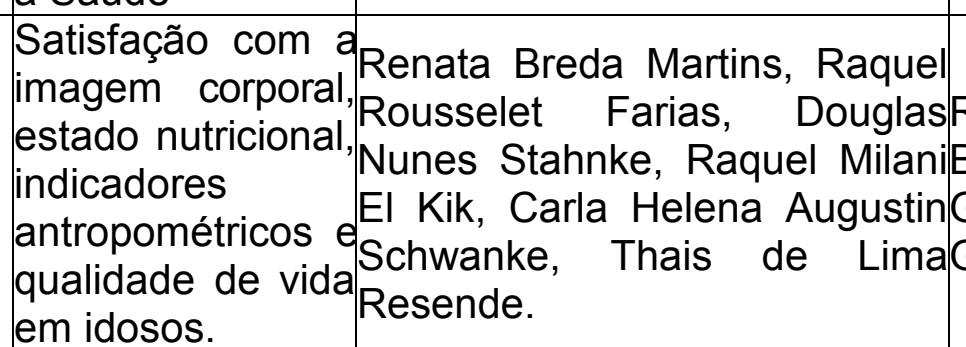 & $\begin{array}{l}\text { Revista } \\
\text { Brasileira } \\
\text { Geriatria } \\
\text { Gerontologia. }\end{array}$ \\
\hline Brasil, 2016. & 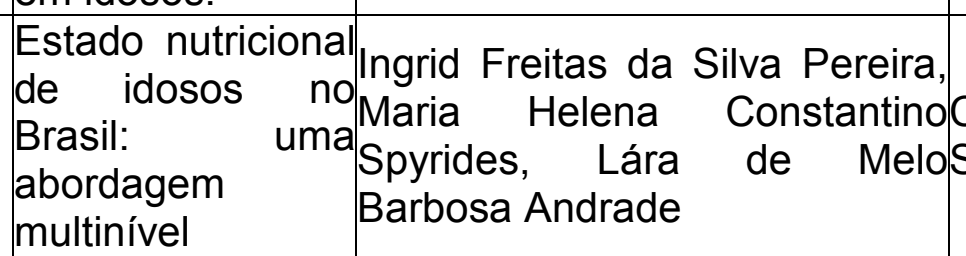 & $\begin{array}{l}\text { Caderno } \\
\text { Saúde Pública. }\end{array}$ \\
\hline Brasil, 2017 & 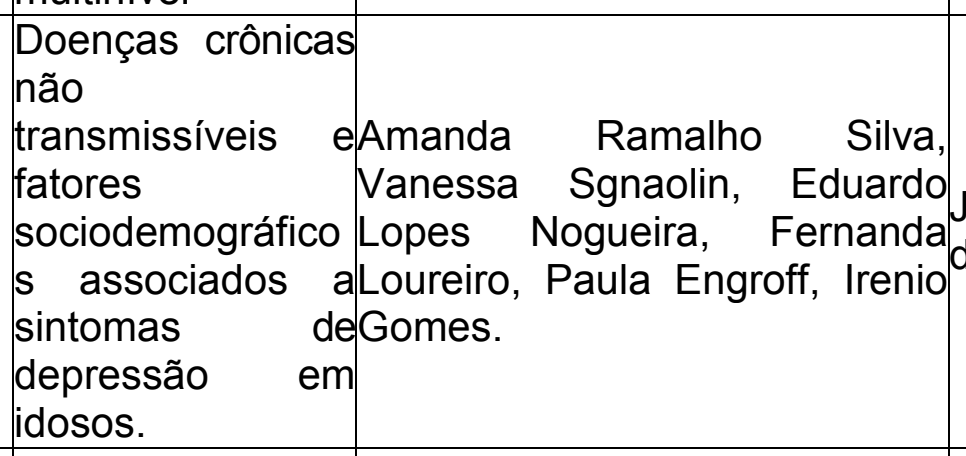 & $\begin{array}{l}\text { Jornal Brasileil } \\
\text { de Psiquiatria. }\end{array}$ \\
\hline Brasil, 2018. & \begin{tabular}{l|l} 
Fatores & Aurora Esteve-Clavero, AnaF \\
associados à Ayora- Folch, Loreto Maciá-F \\
qualidade de vida \\
dos idosos.
\end{tabular} & $\begin{array}{l}\text { Revista Actá } \\
\text { Paulista } \\
\text { Enfermagem. }\end{array}$ \\
\hline
\end{tabular}




\section{DISCUSSÃO}

É indubitável, do ponto de vista social, que o idoso encontra-se numa situação de sucessivas perdas, quer seja do suporte familiar, do status ocupacional, o declínio somático e psíquico e a maior frequência de doenças crônicas e uso de medicamentos, que levam à sentimentos de desânimo e tristeza, gerando quadros depressivos.

Todos os estudos apontam maior prevalência de depressão no público feminino, com idade igual ou superior a 60 anos, na maioria das vezes de classe social baixa, e com um nível de escolaridade inferior, e, em alguns casos analfabetismo. É perceptível que os índices de depressão ficam mais aparentes nos idosos dependentes funcionais, que tiveram a autonomia reduzida, e a depressão pôde ser associada a um envelhecimento de baixa qualidade.

As queixas e demandas mais frequentes, entre as populações observadas foram o abandono, a negligência familiar, a ausência de visitas, a falta de seus pertences e a atenção minimizada. Com isso, surgem as ideações suicidas e até a tentativa do ato, relatados principalmente por pessoas institucionalizadas que não recebem nenhum tipo de visita. Os idosos do sexo masculino que possui uma atividade remunerada possuem uma prevalência de sintomas depressivos menor que os que não exercem nenhuma atividade.

Alguns estudos apontaram associação positiva entre as atividades religiosas e a sensação de bem-estar, de modo que aqueles que estão engajados nestas atividades apresentaram melhor qualidade de vida.

Dentre a presença de doenças crônicas, destacou-se, nos trabalhos analisados a presença de hipertensão arterial, diabetes mellitus, problemas musculoesqueléticos e alterações visuais. Vale salientar que a depressão evidencia os transtornos causados por essas doenças, dificultando a adesão à terapêutica e, por consequência, a recuperação destes pacientes.

Com relação ao consumo de medicamentos, torna-se interessante destacar a real necessidade do consumo de medicamentos em decorrência do adoecimento 
que acomete o idoso e a utilização excessiva de medicamentos para atenuar reações psíquicas geradas por acontecimentos sociais, que podem interagir entre si e potencializar quadros clínicos depressivos. Torna-se necessária a intervenção da equipe multiprofissional para a redução do uso de medicamentos e o estímulo a adoção de práticas saudáveis, tornando o processo de envelhecer bem sucedido.

A situação socioeconômica exerce grande influência no que tange a qualidade de vida no envelhecimento. Variações de renda, condições de moradia e acesso à bens e serviços interferem no acesso e variedade dos alimentos, afetando as escolhas alimentares, interferindo no padrão dietético e alimentar ao longo da vida e refletindo no ganho ou perda de peso e no estado nutricional geral na velhice.

As alterações nutricionais também são asssociadas com diversas complicações, a exemplo do surgimento de lesões por pressão, aumento da prevalência de pneumonia e infecções associadas, redução da capacidade mental e cognitiva, além do número de hospitalizações.

Ainda, é possível entender que, entre os diversos aspectos permeiam o cotidiano do idoso, a redução da autonomia e seus efeitos nas atividades da vida mostram-se mais evidentes, chegando a comprometer o laço social estabelecido durante toda a vida. Além desse aspecto, o abandono familiar é um dos fatores que mais fragilizam interesse pela vida e desencadeia angústias e de sentimentos deprimentes. Assim, a exclusão do âmbito familiar promove fortes impactos na vida do idoso, impactos esses que a longo prazo se tornam irreversíveis.

Nos diversos aspectos que permeiam o processo de envelhecer, a saúde torna-se elemento chave pelo forte impacto que exerce sobre a qualidade de vida. Esta torna-se um grande estigma de preconceitos em relação ao envelhecer e atrai uma representação negativa acerca dessa fase.

Ao identificar um quadro depressivo, a equipe multiprofissional deve trabalhar em conjunto para que a terapêutica mais efetiva seja estabelecida. Desse modo, entende- se que o tratamento não seja restrito ao uso de medicamentos, mas que a escuta e a interação sejam ferramentas chave para a manutenção do estado geral e a redução de sintomas, tornando o idoso sempre protagonista do seu cuidado. 


\section{CONSIDERAÇÕES FINAIS}

Embora o envelhecimento seja uma fase facilmente acometida por diversos transtornos considerados típicos, faz-se necessário uma revisão multidisciplinar das particularidades de cada idoso no seu contexto de assistência à saúde, visando identificar as reais razões dos quadros depressivos. Também, faz-se necessário problematizar se a causa dos sentimentos depressivos e faz paralelo com as patologias que o idoso comumente apresenta.

O impacto da depressão na qualidade de vida e saúde dos idosos interfere no estado nutricional e no padrão medicamentoso utilizado por eles, debilitando ainda mais seu estado geral e gerando situações de dependência e incapacidade, evidenciando sintomas depressivos.

Atitudes simples como reunir-se ao idoso para as refeições, fracionar o consumo de alimentos, estabelecer horários e rotinas, combinar o uso de medicamentos para evitar interações e polifarmácia são medidas simples que, além de melhorar a qualidade de vida do idoso, influencia positivamente na experiência do envelhecimento ativo e com qualidade.

Dessa forma, torna-se necessária a realização de estudos quali-quantitativos que evidenciem essa relação e permitam intervenções efetivas sobre o manejo multiprofissional e a qualidade de vida do idoso, levando em consideração a realidade local, visando a melhoria do estado geral de saúde da pessoa idosa. 


\section{REFERÊNCIAS BIBLIOGRÁFICAS}

ABI-ACKEL, Mariza Miranda et al. Uso de psicofármacos entre idosos residentes em comunidade: prevalência e fatores associados. Revista Brasileira de Epidemiologia, v. 20, p. 57-69, 2017.

ACUÑA, Kátia; CRUZ, Thomaz. Avaliação do estado nutricional de adultos e idosos e situação nutricional da população brasileira. Arquivos Brasileiros de Endocrinologia \& Metabologia, v. 48, n. 3, p. 345-361, 2004.

CROSS, Amanda J. et al. Stakeholder perspectives on pharmacist involvement in a memory clinic to review patients' medication management and assist with deprescribing. Research in Social and Administrative Pharmacy, 2019.

DEKKERS, B. G. J. et al. Invasive candidiasis in the elderly: considerations for drug therapy. Drugs \& aging, v. 35, n. 9, p. 781-789, 2018.

DONIS, A. C. G.; OLIVEIRA, H. S. B.; SOUSA, J. R. P. Prescrição medicamentosa potencialmente inapropriada (PMPI): aplicação dos critérios de Beers 2015 em um grupo de idosos do setor suplementar de saúde [monografia]. São Paulo: Centro Universitário São Camilo, 2017.

GARCIA, A.N.M; ROMANI, S.A.M; LIRA, P.I.C. Indicadores antropométricos na avaliação nutricional de idosos: um estudo comparativo. Revista de Nutrição, Campinas, n. 20, v. 4, p. 371- 378, 2007.

GERMAN, L. et al. Depressive symptoms and risk for malnutrition among hospitalized elderly people. The Journal of Nutrition Health and Aging, v. 12, n. 5, p. 313, 2008.

GUEDES, A. C. B; GAMA, C. R; TIUSSI, A. C. R. Avaliação nutricional subjetiva do idoso: Avaliação Subjetiva Global (ASG) versus Mini Avaliação Nutricional(MAN®). Brasília, nov. 2008.

HAJJAR, Rosmarie et al. Sintomas depressivos e fatores associados em idosos na Atenção Primária à Saúde. Revista da Rede de Enfermagem do Nordeste, v. 18, n. 6, p. 727-733, 2017.

LANGA, Kenneth $M$. et al. Extent and cost of informal caregiving for older Americans with symptoms of depression. American Journal of Psychiatry, v. 161, n. 5, p. 857- 863, 2004.

MARTINS, R. B. et al. Satisfação com a imagem corporal, estado nutricional, indicadores antropométricos e qualidade de vida em idosos. Revista Brasileira de Geriatria e Gerontologia, Rio de Janeiro, v. 21, n. 6, p. 667-679, 2018.

NÓBREGA, Otávio de Tolêdo; KARNIKOWSKI, Margô Gomes de Oliveira. A terapia medicamentosa no idoso: cuidados na medicação. Ciência \& saúde coletiva, v. 10, p. 309-313, 2005.

PANCOTE, Camila Garcel et al. Envelhecimento e uso de psicotrópicos. Enfermagem Brasil, v. 17, n. 5 , p. 426-427, 2018.

PEREIRA, Ingrid Freitas da Silva; SPYRIDES, Maria Helena Constantino; ANDRADE, Lára de Melo Barbosa. Estado nutricional de idosos no Brasil: uma abordagem multinível. Cadernos de Saúde Pública, v. 32, p. e00178814, 2016. 
QUINALHA, Juliana Vasconcelos; CORRER, Cassyano Januário. Instrumentos para avaliação da farmacoterapia do idoso: uma revisão. Revista brasileira de geriatria e gerontologia, $v$. 13, n. 3, p. 487-499, 2010.

SECOLI, Silvia Regina. Polifarmácia: interações e reações adversas no uso de medicamentos por idosos. Rev. bras. enferm., Brasília , v. 63, n. 1, p. 136- 140, Feb. 2010.

SILVA, Amanda Ramalho et al. Doenças crônicas não transmissíveis e fatores sociodemográficos associados a sintomas de depressão em idosos. J Bras Psiquiatr, v. 66, n. 1, p. 45-51, 2017.

VERA, Elaine Cristina Biffi Alonso et al. Terapia medicamentosa do idoso: fatores de influência. 2017.

YOSHIMURA, Kazuya et al. Relationship between depression and risk of malnutrition among community-dwelling young-old and old-old elderly people. Aging \& mental health, v. 17, n. 4, p. 456-460, 2013.

WORLD HEALTH ORGANIZATION et al. Envelhecimento ativo: uma política de saúde. 2005. 